\title{
Unilateral Internuclear Ophthalmoplegia Due to Microvascular Pontine Infarction
}

\author{
*S Hossain ${ }^{1}$, T Guda ${ }^{2}$, F Chowdhury ${ }^{3}$, S Hossain ${ }^{4}$
}

\begin{abstract}
Internuclear Ophthalmoplegia (INO) is a clinical syndrome which develops due to a lesion in the medial longitudinal fasciculus (MLF). It is a horizontal gaze disorder characterized by impaired adduction of eye on the side of the lesion of medial longitudinal fasciculus with dissociated nystagmus of the opposite abducting eye. This lesion is mostly caused by multiple sclerosis (usually bilateral lesion), stroke and tumours. A case with unilateral internuclear ophthalmoplegia due to vascular lesion was admitted in Anwer Khan Modern Medical College \& Hospital on 27/05/2017.
\end{abstract}

Key Words: Internuclear Ophthalmoplegia (INO), Medial longitudinal fasciculus (MLF), impaired adduction, pontine infarction, nystagmus, diplopia.

\section{Introduction}

Internuclear ophthalmoplegia is a horizontal gaze disorder of eye movement, classically characterized by impaired adduction of the eye on the side of a lesion involving the medial longitudinal fasciculus with dissociated nystagmus of the opposite abducting eye. ${ }^{1,2}$ This limited adduction of the eyes leads to horizontal diplopia. Convergence is generally preserved if the lesion involve the anterior part of MLF and is discrete but impaired if the lesion involve the posterior part or extensive. ${ }^{8,9}$ In young patients, this syndrome is most commonly caused by multiple sclerosis. ${ }^{1,3,6}$ In fact, INO is the most common oculomotor manifestation of multiple sclerosis. ${ }^{3}$ The same eye-movement disorder in an older age group is usually attributed to cerebrovascular disease. ${ }^{7}$ The interneurons of the MLF are intermixed with the abducens nerve nucleus, which lies dorsally in the pons near the genu of the seventh cranial nerve. A lesion in the facial coliculus produces a combination of INO and facial palsy. Interestitial nucleus of Cazol in the mid brain situated between $3^{\text {rd }}$ nerve nucleus and superior colliculus is the vertical gaze center which is connected to the vestibular nucleus in the pons by MLF.

Here we reported a middle aged patient who suffered from pontine infarction showing unilateral INO with horizontal nystagmus and normal convergence. But vertical or torsional nystagmus were absent. His reflex facial and body movement due to visual stimulation were normal. This may prove that the lesion only involve the anterior part of LMF but not the paramedian tract (PMT) neurons or ventral tegmental tract (VTT) responsible for vertical nystagmus. LMF that connect the vestibular nucleus with interstitial nucleus of Cazol which are probably act as a neural integrator for vertical gaze and torsional eye movement as well as reflex face movement due to visual stimulation ${ }^{8,9}$ is also unaffected. Reflex body movement due to visual stimulation were also normal indicate normal function of tectospinal tract. To our best knowledge, only a few cases have been reported presenting INO with vertical or torsional nystagmus in human. ${ }^{2}$

\footnotetext{
$1 *$ Prof. Dr. Sanwar Hossain, Professor \& Head, Department of Ophthalmology, Anwer Khan Modern Medical College, Dhaka, Bangladesh

${ }^{2}$ Dr. Titus Leonard Guda, Registrar, Department of Ophthalmology, Anwer Khan Modern Medical College, Dhaka, Bangladesh

${ }^{3}$ Dr. Forhad Chowdhury, Medical officer, Department of Ophthalmology, Anwer Khan Modern Medical College Hospital, Dhaka, Bangladesh

${ }^{4} \mathrm{Dr}$. Md. Somir Hossain, Honorary Medical Officer, NIO \&H

*Corresponding author

Date of submission: 13.06.2017, Date of acceptance: 21.08.2017
}

AKMMC J 2018; 9(1) : 71-74 


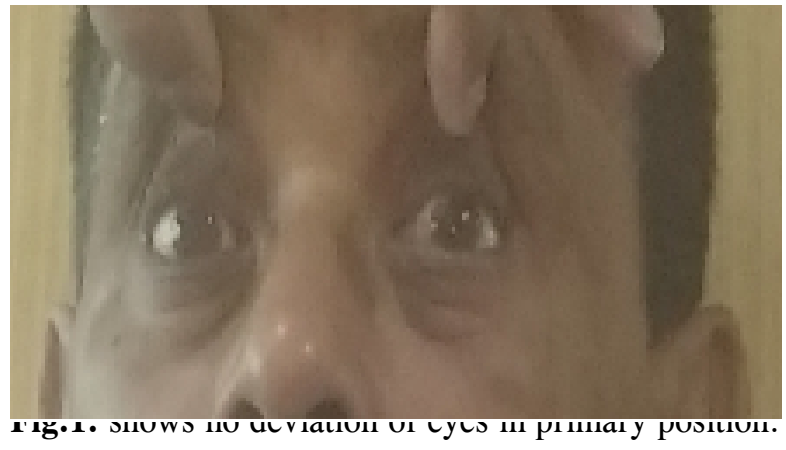

Case history

Nandolal Mojumder 60 years old male, businessman hypertensive, diabetic, non asthmatic and associated with IHD (PCI with 1 stenting) got admitted in this hospital on $27 / 5 / 17$ with the complaints of tingling and numbness of right upper limb and left side of face for 3 days with blurring of vision in the right eye and double vision for same duration. He also gave history of falling down 3 days back at the beginning of attack . There was no history of fever, nausea, vomiting and vertigo. His bowel and bladder habits were normal.

On general examination, patient was irritable, ill looking, non anemic, acyanotic, non icteric, decubitus on choice. His pulse was 68 beat $/ \mathrm{m}$, blood pressure $130 / 80 \mathrm{mmHg}$, respiratory rate was $16 / \mathrm{min}$.

On nervous system examination, he was oriented, GCS $15 / 15$. Motor function and Sensory functions were intact. Cerebellar system examination was normal and there was no sign of meningeal irritation.

Ocular examination revealed an adduction deficit in the left eye (Fig.2) and as expected horizental nystagmus in the right eye on right gaze movement.

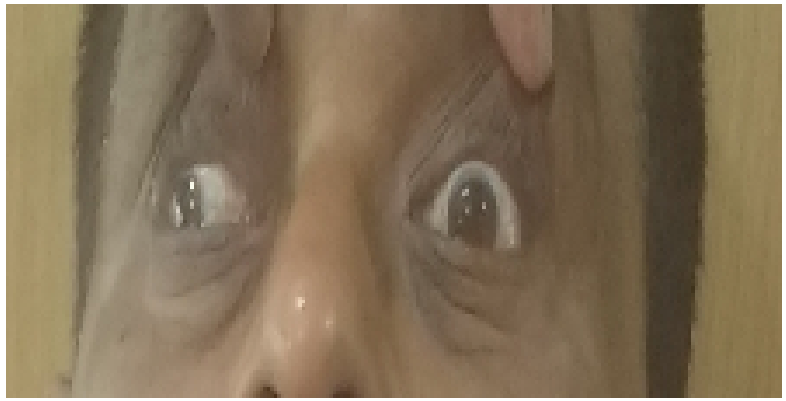

Fig.-2: Adduction deficit in the left eye Note worthily, no bilateral or unilateral vertical nystagmus or torsional nystagmus was observed. Convergence movement was normal (Fig.3). His left gaze movement is also normal (Fig.4). Reflex face and body movement on visual stimulation was normal.

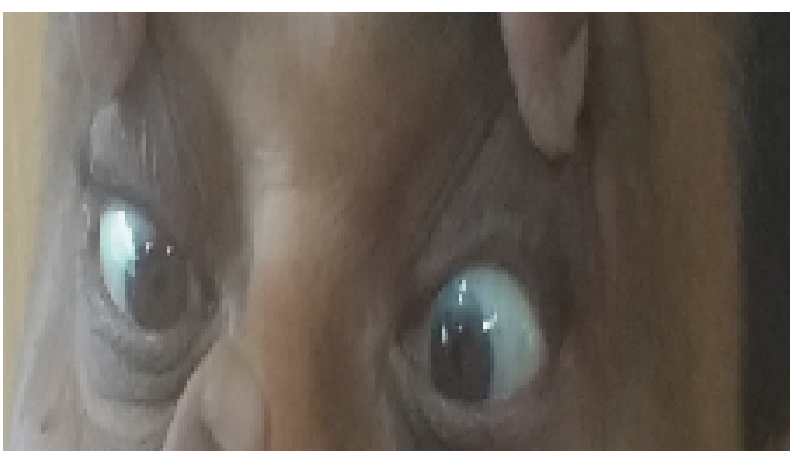

Fig.-3: Normal convergence movement.

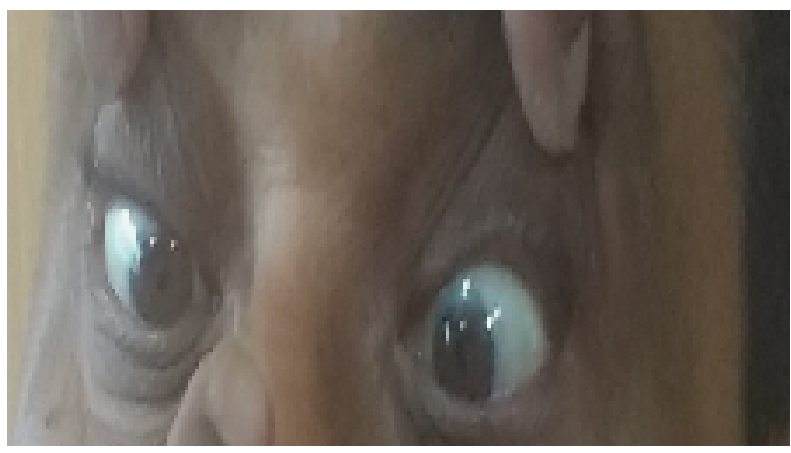

Fig.-4: Normal left gaze movement

On ocular examination following significant findings were found:

\begin{tabular}{|c|c|c|}
\hline & Right eye & Left eye \\
\hline $\begin{array}{l}\text { Visual acuity } \\
\text { (BCVA) }\end{array}$ & $\begin{array}{l}6 / 18 \text { without glass } \\
\text { and } 6 / 6 \text { with glass }\end{array}$ & $\begin{array}{l}6 / 9 \text { without glass and } \\
6 / 6 \text { with glass }\end{array}$ \\
\hline Ocular motility & $\begin{array}{l}\text { Full in all gazes. } \\
\text { Nystagmus } \\
\text { present in right } \\
\text { gaze movement }\end{array}$ & $\begin{array}{l}\text { Impaired adduction in } \\
\text { right gaze movement } \\
\text { (dextroversion) }\end{array}$ \\
\hline $\begin{array}{l}\text { Convergence } \\
\text { movement }\end{array}$ & $\begin{array}{l}\text { (dextroversion). } \\
\text { Normal }\end{array}$ & Normal \\
\hline $\begin{array}{l}\text { Torsional and } \\
\text { vertical movement }\end{array}$ & No nystagmus & No nystagmus \\
\hline $\begin{array}{l}7 \text { th and 6th nerve } \\
\text { function }\end{array}$ & normal & normal \\
\hline $\begin{array}{l}\text { Anterior segment } \\
\text { Pupil }\end{array}$ & $\begin{array}{l}\text { Normal } \\
\text { Normal }\end{array}$ & $\begin{array}{l}\text { Normal } \\
\text { Pin pointed }\end{array}$ \\
\hline Fundus & Normal & Normal \\
\hline
\end{tabular}


Unilateral Internuclear Pphthalmoplegia Due to Microvascular Pontine

Other systemic examination reveals no abnormalities.

On laboratory examination, complete blood count shows no abnormality. Bleeding time, clotting time, Prothrombin time, APTT were normal. Random blood sugar: $13.5 \mathrm{mmol} / 1$. Serum electrolyte shows Sodium $131 \mathrm{mmol} / 1$. SGPT: $22 \mathrm{U} / 1$. Serum lipid profile normal. Serum creatinine $1.2 \mathrm{mg} / \mathrm{dl}$. Hbs Ag, HIV Ab, Anti HCV ab \& VDRL all were negative. Urine routine \& microscopic examination shows ketone bodies $(+++)$. ECG was normal. Echocardiography: EF $70 \%$.

We had done a specific investigation MRI of Brain showing acute microvascular infarct in the pons. [Fig.5].

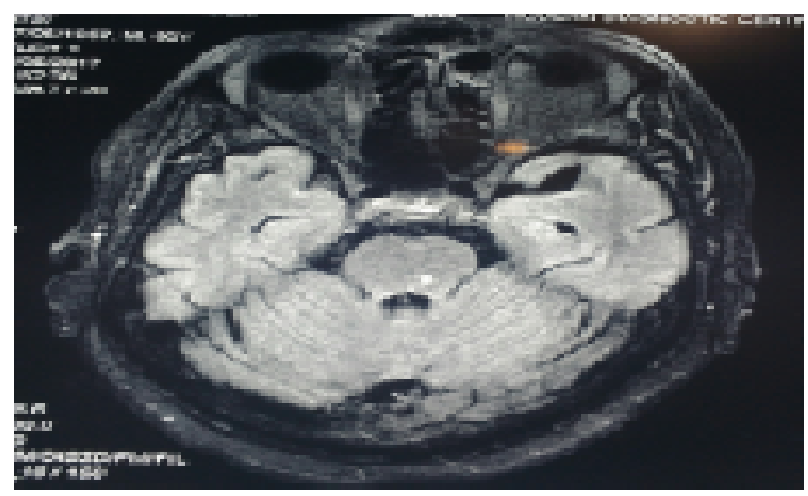

Fig.-5: Microvascular infarct in the Pons.

His numbness and tingling sensation of limbs and face disappeared when he was discharged from hospital 11 days after onset of symptom. But the left INO and abduction nystagmus in right eye were still present.

\section{Discussion}

The term internuclear lesion denotes lesion localization between the nerve III and VI nuclei. MLF a tract which lies in the tegmentum of the brainstem connects the third and sixth nerve nuclei and also connects both these nuclei with the vestibular nuclei. Interestitial nucleus of Cazol also remain connected with vestibular nucleus via MLF essential for torsional eye movement. Pontine lesions like, INO, paramedian pontine reticular formation (PPRF) syndrome, abduction paralysis or one-and-a-half syndrome may result in horizontal eye movement disorders. PPRF at the level of abducens nucleus serves as the center for horizontal gaze eye movement. Instruction of PPRF for horizontal gaze movement is achieved by the simultaneous innervations of the ipsilateral lateral rectus and the contralateral medial rectus via MLF. In this case Interruption of the MLF of left side at the midpons causes a discrete adduction deficit of the left eye a sign called INO. Abduction dissociated nystagmus occurs in the contra lesional right eye during horizontal gaze movement. It has been hypothesized to reflect impaired inhibition in the medial rectus muscle of the abducting eye. Clinically, when testing the range of ocular movement, the syndrome presents with diplopia in right horizontal gaze movement, which is not present on looking straight forward and nystagmus of the right eye in abduction. ${ }^{2}$ In young individuals, bilateral INO quite commonly develops in association with multiple sclerosis due to MLF demyelination. But in this case, the involvement is only left MLF and other positive brain findings of MS in MRI are absent. MRI revealed a small pontomesencephalic paramedial lesion. Sudden falling down at the onset of attack may be due to involvement of autonomic nervous system. Same involvement is also responsible for miosis in the left eye. The numbness and tingling sensation of right upper limb and left side of face of this patient on admission might be due to temporary involvement of left corticospinal tract and left facial colliculus. But the problems were absent 11days after onset of attack at the time of discharge from the hospital. MRI shows a tiny lesion in the MLF anterior to the floor of $4^{\text {th }}$ ventricle. The lesion do not involve the PMT which lies just lateral to the MLF responsible for vertical nystagmus or do not involve the adjacent 6th nerve nucleus. There was temporary involvement of left facial colliculus which lead to left facial numbness and tingling sensation. This patient could change and maintain his reflex postural movement of head and body in response to visual stimuli, indicate his tectospinal tract or vestibulo cerebellar tract or the part of MLF that connect the vestibular nucleus with the interstitial nucleus of Cazol were unaffected. The anatomical location of 
tectospinal tract is just anterior to MLF. So we can conclude that the part of brain stem other than MLF responsible for horizontal gaze as well as vertical gaze and torsional eye movement like PPRF, PMT, VTT and the part of MLF that connect the vestibular nucleus with the interstitial nucleus of Cazol, those participate as oculo motor integrator ${ }^{1,3,4}$ were unaffected. It is noteworthy that the interstitial nucleus of Cazol (INC) situated between the red nucleus and the superior colliculus of mid brain is the neural integrator for vertical gaze holding. It receives disynaptic potentials through the ipsilateral and contralateral MLF. In bilateral MLF lesion would involve the function of this vertical integrator. ${ }^{3,5}$

\section{Conclusion}

The occurrence of unilateral INO is very rare especially in our country as it usually appears as bilateral and common cause is multiple sclerosis. The general practitioners / primary care physician, neurologist, ophthalmologist/neuro-ophthalmologist and other health professionals should all be involved in the management and follow up of patients with INO.

\section{Interest of conflict: None}

\section{References}

1. Lee SC, Lee SH, Lee KY, et al. Transient upbeat nystagmus due to unilateral focal pontine infarction. J Clin Neurosci 2009; 563-565.
2. Srivastava AK, Tripathi M, Gaikwad SB, et al. Internuclear ophthalmoplegia and torsional nystagmus: an MRI correlate. Neurol India 2003; 51: 271-272.

3. Ranalli PJ and Sharpe JA. Vertical vestibuloocular reflex, smooth pursuit and eye-head tracking dysfunction in internuclear ophthalmoplegia. Brain 1988; 111: 1299-1317.

4. Nakamagoe K, Iwamoto $\mathrm{Y}$ and Yoshida K. Evidence for brainstem structures participat-ing in oculomotor integration. Science 2000;857859.

5. King WM, Precht W and Dieringer N. Synaptic organization of frontal eye field and vestibular afferents to interstitial nucleus of Cajal in the cat. J Neurophysiol 1980; 43: 912-928.

6. Pierrot-Deseilligny $\mathrm{C}$ and Milea D. Vertical nystagmus: clinical facts and hypotheses. Brain 2005; 128: 1237-1246.

7. Pierrot-Deseilligny C, Milea D, Sirmai J, et al. Upbeat nystagmus due to a small pontine lesion: evidence for the existence of a crossing ventral tegmental tract. Eur Neurol 2005; 54:186-190.

8. Buttner-Ennever JA and Horn AK. Pathways from cell groups of the paramedian tracts to the floccular region. Ann N Y Acad Sci 1996;532-540.

9. Lee H, Yi HA and Kim HA. Do the paramedian tract neurons in pons take a role as a vertical neural integrator in humans? J Neurol Sci 2012; 321: 107-110. 